\title{
Organización y gestión del diseño de sistemas de información
}

\author{
Jesús Tramullas Saz \\ Universidad de Zaragoza \\ Departamento de Ciencias de la Documentación \\ e Historia de la Ciencia
}

\subsection{Resumen}

Este trabajo presenta una revisión de los procesos de gestión y control del desarrollo de los sistemas de información. Realiza una análisis del concepto de sistema de información, en el contexto de una organización, las fases principales del proceso de diseño y creación del mismo, e indica las herramientas más comunes que permiten controlar el desarrollo del proyecto, de forma que cumpla con las expectativas generadas.

Palabras clave: Sistemas de Información: Desarrollo e Implementación. Mecanismos y Herramientas de Control.

\subsection{Abstract}

This paper reviews the control and management process in information systems design and development. The concept of information systems is analyzed form a managerial point of view. The main design and implementation stages and the most common tools for project controlling are considered.

Keywords: Information systems: design and implementation. Control mechanism and tools.

\section{Introducción.}

La gestión y la planificación del diseño y desarrollo de un sistema de información suelen ser tareas que no han recibido, en el entorno de las ciencias de la información y la documentación, y tal y como revela la bibliografía, todo el interés que merecen, favoreciendo, por contra, los aspectos relacionados con el diseño e implantación del sistema (Rowley, 1994). La aparente "dejación" de estas tareas tiene una estrecha relación con la falta de formación sobre estas cuestiones, resultado de la despreocupación mostrada por temarios de oposiciones y, 
hasta fecha relativamente reciente, por planes de estudios universitarios basados en una concepción excesivamente "tradicional" del trabajo del bibliotecario/documentalista.

La adquisición de la tecnología para soportar un sistema de información resulta sencilla. El desafío consiste en adecuar la explotación de la tecnología a las metas de la organización en la que se implanta.

En cambio, en el momento actual, es corriente escuchar en los más diversos foros términos como "gestión de centros", "gestión y control de calidad", "planificación operativa" y otros del mismo tipo, que invariablemente están haciendo referencia a técnicas y principios del management, es decir, a la gestión de organizaciones y de proyectos. Gestión ésta que se resume en dos palabras: dirección y coordinación, pudiendo especificar dentro de la dirección las tareas de planificación, organización, control, coordinación y mando.

El correcto desempeño de ambas tareas resulta crucial, y factor determinante de éxito, en el diseño e implantación de cualquier sistema de información, sea del tipo que sea. Se trataría, entonces, de planificar, dirigir, controlar la realización y analizar los resultados de todos los trabajos relacionados con el diseño e implantación del sistema de información. Si se aplican estos principios a un proceso de informatización de una unidad de información, no bastaría con preguntar, evaluar y poner en marcha. Un enfoque riguroso que ofreciese garantías de éxito, calidad al fin y al cabo, prestaría tanta importancia a estas cuestiones como a las relacionadas con el proceso de dirección y coordinación entre todas ellas, así como a cuestiones colaterales de suma importancia, que de otra forma pasarían inadvertidas. A tal fin han insistido Vickery y Vickery (1992: 210):

"The practical work of information scientifist is concerned with development, operation and management of information systems of all kinds, and with the various techniques and technologies by which information is transferred."

\section{Organización, sistema, dirección}

El tratamiento de las entidades de tipo organizativo resulta un lugar común en la bibliografía especializada desde comienzos del presente siglo. A los fines de este trabajo, definiremos la organización como la coordinación racional de las actividades de un grupo de personas con el propósito de lograr algún fin (Lucas, 1987: 29). Las organizaciones pueden mostrar diferentes tipos y estructuras, generalmente dependientes de los objetivos y cultura interna de la propia organización. Si es necesario puntualizar que en toda organización se superponen niveles de organización formales e informales. Además, en todas las organizaciones, independientemente de la tipología a la que respondan, se identifican los principios de incertidumbre, especialización, coordinación e interdependencia. 
El segundo elemento a considerar es el sistema de información, término que en la actualidad resulta polisémico en grado sumo, ya que se utiliza para designar a entidades muy diversas. Siguiendo a Lucas, un sistema de información es “... un conjunto de procedimientos organizados que, cuando se ejecutan, proporcionan información para la toma de decisiones y/o el control de la organización." (1987: 16). Tomando en consideración las características de los sistemas y las aportaciones de otros investigadores, Miguel y Piattini proponen la siguiente aproximación (1993: 17):

“... definimos el sistema de información como un conjunto de elementos ordenadamente relacionados entre sí de acuerdo con unas ciertas reglas que aporta al sistema objeto (es decir, a la organización a la cual sirve y que le marca las directrices de funcionamiento) la información necesaria para el cumplimiento de sus fines, para lo cual tendrá que recoger, procesar y almacenar datos, procedentes tanto de la misma organización como de fuentes externas, facilitando la recuperación, elaboración y presentación de los mismos."

El tercer elemento a tratar se engloba bajo el término dirección, dirección que, por supuesto, se referirá a los sistemas de información. Siguiendo nuevamente a Lucas (1987: 18),

"La dirección del sistema consiste en actividades orientadas al planeamiento y control. El planeamiento abarca establecer metas, utilizar recursos y desarrollar un programa para hacerse cargo de diferentes actividades y una estrategia para entenderse con el medio ambiente. El control se encarga de la ejecución de los planes. El flujo de información y realimentación está asociado con el control, de tal modo que un sistema pueda evaluar sus propios planes."

En toda organización pueden detectarse tres niveles de gestión: un nivel elevado, de alta gestión, orientada a la estrategia de la organización a largo plazo; un nivel medio, táctico, a medio plazo, y un nivel básico, de primera línea, orientada a la operación diaria.

Desde una perspectiva sistémica, una organización es un sistema abierto, y este sistema posee, como todos los sistemas, un sistema de información. Este cumple la función de sistema nervioso, que interrelaciona todos los componentes de la organización. Ambos son sistemas dinámicos, poseen elementos e interacciones entre esos elementos, y en ocasiones el sistema de información está pasando a ser no ya un subsistema de la organización, sino el sistema que da sentido a la organización. Esto permite adoptar un enfoque sistémico para la planificación, diseño e implantación de un sistema de información. Como acertadamente ha señalado Checkland (1993: 19),

"Lo que distingue a los sistemas es que, en sí, son una materia que puede hacer referencia acerca de otros temas... es una metadisciplina, cuyo material sustancial se puede aplicar virtualmente dentro de cualquier otra disciplina."

Scire. 2 : 2 (jul.-dic. 1996) 121-131 
La metodología de sistemas permite tres posibles aproximaciones:

1. Estática: analiza la estructura básica, los subsistemas y sus elementos, la comunicación y sus canales.

2. Dinámica: analiza las funciones del sistema y de los subsistemas, las relaciones con otros sistemas, con suprasistemas y ecosistemas, y la homeóstasis entre ellos.

3. Dialéctica: estudia la reproducción de los sistemas, sus mutaciones y transformaciones estructurales, la autoorganización y la emergencia.

\section{Los sistemas de información desde la perspectiva de la organi- zación}

Son numerosos los intentos por conceptualizar y definir los sistemas de información. Independientemente de las numerosas aproximaciones (Tramullas, en prensa), Martínez López (1995, p. 32-56) ha realizado recientemente una revisión de los diferentes enfoques que un sistema de información puede tener en una organización, y de cómo ese enfoque influye, a su vez, en las metodologías de desarrollo utilizadas en su creación, y en la explotación de los mismos. Este autor diferencia entre:

1. Aquéllos que ponen el énfasis en los medios tecnológicos de soporte.

2. Aquéllos que se centran en la información.

3. Los que consideran los SI como subsistemas del sistema total de la organización.

4. Los que toman a la organización como un sistema de información.

5. Aquéllos que utilizan los SI como modelos para la propia organización.

Como consecuencia del enfoque adoptado, varían las metodologías de desarrollo, y, por ende, los mecanismos de planificación y control aplicados. El enfoque más aceptado, en la actualidad, es el correspondiente al punto 5, que facilita la integración progresiva de la organización y de sus recursos informativos. En este entorno debe comprenderse el auge que ha adquirido en los últimos años la IRM (Information Resources Management, o Gestión de Recursos de Información) (Cornellá, 1994). Se trata de "organizar para la información", por lo que el propio diseño de los sistemas debe orientarse en este sentido, más que en las perspectivas organizativas o tecnológicas. Sin embargo, no puede dejar de notarse que los SI diseñados tienen su reflejo más evidente en el portafolio de aplicaciones. 


\subsection{EI portafolio de aplicaciones}

El sistema de información se contextualiza en una organización que tiene unas metas y unos objetivos. Estas metas y objetivos deben ser necesariamente consideradas en la planificación de sistemas de información, ya que serán las que fijen las prioridades en el diseño de los mismos. Se trata de obtener una visión del impacto de estos sistemas en la organización, de forma integrada y complementaria a la misma.

El conjunto de sistemas de información que se plasman en aplicaciones informáticas recibe el nombre de portafolio de aplicaciones. Los sistemas o aplicaciones deben tender a ofrecer cuatro niveles de integración (Senn, 1992, p. 62):

1. Horizontal: relaciones ente áreas funcionales de la organización.

2. Vertical: relaciones jerárquicas correspondientes con las existentes en la organización.

3. Física: relaciones a nivel geográfico con otras localizaciones de la organización.

4. Del medio externo al interno: relaciones con proveedores y con clientes externos.

\section{El planteamiento del proyecto}

\subsection{Existencia de necesidades: identificación y propuesta del sistema}

El diseño de un sistema de información responde a la expresión de una necesidad en una organización, necesidad que sólo puede ser cubierta mediante el diseño, desarrollo y puesta en marcha de un sistema cuyo objeto de trabajo sea información (Senn, 1992:, p.84-85; Burch y Grudnitsky, 1992). Para el tratamiento de estas necesidades se han propuesto varias metodologías:

1. Planeación de sistemas empresariales (BSP): desarrollado por IBM, se basa en la identificación de los datos necesarios para poner en marcha una organización.

2. Planeación estratégica de arquitectura de computadoras de Nolan, Norton \& Co.: se trata de desarrollar una potente infraestructura técnica que sirva como base para el desarrollo de futuros sistemas.

3. Factores críticos de éxito (CSF): intenta identificar las áreas que son claves para la organización e incorporarlas a los sistemas de información de la misma.

La propuesta de diseño e implantación de un nuevo o remozado sistema de información conlleva la realización de un estudio preliminar, que determine el beneficio que puede obtener la organización con el nuevo sistema. No se trata de 
un análisis detallado de requerimientos, sino de una evaluación previa que determine la factibilidad (operacional, técnica y económica) de la propuesta.

\subsection{Un punto de partida: el proyecto tipo estándar}

Como base para la gestión del desarrollo de un proyecto de diseño de sistema de información, se ha propuesto la utilización de un proyecto tipo estándar (Martín Sánchez, 1995, p. 269-282), mediante el diseño de una parrilla que responda a cuestiones de tipo ¿Qué hay que hacer? ¿Quién tiene que hacerlo? ¿Cuando debe hacerse? ¿Cuánto va a costar? y ¿A quién y cómo hay que informar?.

La construcción del proyecto seguirá los siguientes pasos:

1. Introducción de los datos de detalle del proyecto: nombre, descripción, estructura, calendario, tipo de codificación...

2. Especificar las fases, tareas y sus posibles divisiones que componen el proceso de desarrollo del sistema.

3. Determinar el tiempo de realización de las tareas elementales o básicas, al más bajo nivel.

4. Ordenar las tareas según la secuencia de realización, indicando su posible coincidencia o dependencia.

5. Especificar los recursos disponibles.

6. Indicar los costes por recurso, incluyendo costes de personal, de equipamiento, amortizaciones, etc.

7. Por último, la revisión de todo lo anterior.

A la construcción del proyecto siguen las tres etapas clásicas del desarrollo de un proyecto:

1. Planificación: consiste en decidir cómo, cuándo y por quién se van a ejecutar las tareas, definiendo de forma detallada todas y cada una de las actividades a realizar. En casi todas las ocasiones existe un factor impredecible de modificación, atribuible a muy diversas causas, que hace que la retroalimentación de la planificación sea continua y necesaria durante todo el desarrollo del proyecto.

2. Seguimiento: se trata de controlar la evolución de los trabajos, tanto en lo que respecta a tiempos como a recursos. Supone la recogida y estudio de datos según plazos predeterminados, puntos de inflexión, etc.

3. Evaluación: tras el fin del desarrollo, analizar como ha transcurrido el mismo, comparando las estimaciones iniciales con las reales, y extraer las conclusiones pertinentes, con vista a futuros proyectos. 


\subsection{Primera cuestión crucial: la incertidumbre}

En la planificación y el diseño de sistemas de información existe siempre un nivel de incertidumbre. Este factor se encuentra presente en toda la duración e implantación del sistema, y depende de múltiples y variados factores, como la diferente concepción del sistema por parte de los usuarios, las limitaciones imprevistas, económicas o tecnológicas. Una adecuada planificación y gestión del proceso de informatización debe limitar y reducir en lo posible el nivel de incertidumbre.

\subsection{Segunda cuestión crucial: la estimación y control de tiempos}

El desarrollo de un sistema de información tiene uno de sus principales enemigos en el tiempo. La duración temporal de un proyecto es uno de las cuestiones claves para alcanzar los objetivos fijados. La estimación de tiempos puede hacerse tomando como base otras experiencias anteriores, intuitivamente (basado principalmente en la experiencia), o mediante una fórmula matemática estándar que combina varios factores. Al ser el desarrollo de un sistema de información una actividad que en el $90 \%$ de las ocasiones conlleva la creación de una aplicación informática estructurada, se pueden calcular gran parte de los tiempos de forma muy aproximada a la realidad.

Para el establecimiento de un calendario de desarrollo del sistema se utilizan varios métodos de representación gráfica, entre los que deben destacarse (Senn, 1992, p. 882-887; Quang y Gonin, 1994, p. 43-50):

1. Diagramas de barras o de Gantt: consiste en crear una representación de eje de barras, mostrando en el eje vertical las tareas y en el horizontal las unidades de tiempo, representando la duración de cada tarea una barra a lo largo de las unidades de tiempo.

2. Diagrama de eventos críticos: muestran los sucesos críticos del desarrollo y la secuencia en que deben llevarse a cabo.

3. Diagramas de Pert: es el más sofisticado, y fue desarrollado por la U.S. Navy en 1958 con motivo del proyecto de armamento Polaris. Los diagramas de Pert muestran las actividades a desarrollar, su duración temporal y las relaciones existentes entre ellas, a través del uso de nodos y rutas. Al concluir el diagrama, se estudia la ruta crítica, que marcará el tiempo total necesario.

\subsection{El control y retroalimentación del desarrollo: recorridos estructurados}

Por recorrido estructurado se entiende una revisión planificada de un sistema por parte de las personas involucradas en el desarrollo del mismo. Su finalidad es encontrar áreas donde sea posible mejorar el sistema o el desarrollo. Las revisiones pueden centrarse en los requerimientos, diseño, pruebas y cuantos elementos 
se consideren pertinentes, y sus conclusiones y avisos deben considerarse dentro del propio proceso de desarrollo.

\section{6. El aspecto humano: la gestión de personal y grupos de trabajo}

Los proyectos son desarrollados por personas. Estas personas son las que ejecutan las tareas, y son responsables de su correcto resultado en tiempo y forma. La unión y coordinación de estas tareas es una de las funciones claves en la planificación y gestión del desarrollo de sistemas de información. Pueden darse múltiples variaciones en la organización de los grupos de trabajo, pero todas ellas deben coincidir en objetivos, procedimientos y tiempos dentro del proyecto.

\section{El diseño y desarrollo de sistemas de información}

El diseño y desarrollo de sistemas de información sigue una metodología, “...un enfoque para organizar, dirigir y realizar las actividades del ciclo de vida de un sistema de información." (Rodríguez y Márquez, 1993, p. 40). Todas las metodologías muestran tres fases genéricas, correspondientes a (Pablo, 1989, p. 156-189):

1. Planificación preliminar: analizar el problema de información en el contexto organizativo, definir los objetivos del sistema y organizar el proyecto y equipo de trabajo.

2. Fase de diseño preliminar: encontrar una solución a los problemas de información, mediante la recogida de datos, la definición de necesidades y medios, el análisis de diseños alternativos y la selección del diseño definitivo.

3. Fase de desarrollo: diseño de los elementos del sistema, entradas, salidas, procesos, controles, archivos y ficheros.

4. Fase de instalación y desempeño: puesta en marcha y desempeño del sistema.

Desde una perspectiva sistémica, la metodología deberá ofrecer tanto elementos de filosofía ("qué"), como de técnicas ("cómo"), con varias fases de desarrollo (Checkland, 1993, p. 190-208):

1. Expresión del problema.

2. Definiciones raíz de sistemas pertinentes.

3. Confección y verificación de modelos conceptuales: se trata de construir un modelo de sistema de actividad para lograr la transformación descrita en la definición raíz. 
4. Comparación de los modelos conceptuales con la realidad.

5. Habilitación de los cambios deseables y necesarios.

La metodología seleccionada para el diseño y desarrollo dependerá sobremanera de las preferencias de la organización, de su cultura corporativa o de factores preeminentes en contextos específicos. Puede adoptar el enfoque clásico del ciclo de vida, con un desarrollo en cascada, o el enfoque por prototipos, o el modelo evolutivo, o el diseño estructurado... pueden combinarse entre ellos, etc.

Por su importancia, debe citarse el modelo establecido por la escuela de Lancaster, para la cual el punto de partida es considerar quién, en términos de rol, necesita cual información para cual propósito, en un análisis en varias etapas (Wilson, 1993, p. 258-259):

1. Desarrollar una descripción de la actividad de la organización bajo estudio.

2. Derivar las categorías de información requeridas para apoyar las actividades en los modelos y las actividades particulares de las que puede obtenerse esta información.

3. Definir los roles de administración en términos de las actividades para las que cada poseedor de rol tiene la responsabilidad de tomar decisiones.

4. Emplear estas actividades de rol para convertir los flujos de información "actividad a actividad" en flujos de información "rol a rol".

5. Definir los sistemas de información necesarios que corresponden a las necesidades de desempeño de las actividades que cada sistema apoya.

\section{Control del desarrollo}

El proyecto y su metodología son un medio para alcanzar un fin, un objetivo concreto. Por lo tanto, resulta de suma importancia todo el proceso de planificación del proyecto, así como el control del desarrollo de las tareas pertinentes, en los periodos de tiempo establecidos. Se trata, entonces, de alcanzar el objetivo previsto en el tiempo previsto, con el coste justo y la calidad necesaria (Quang y Gonin, 1994: 81-89), intentando limitar, en lo posible, los factores de incertidumbre vistos con anterioridad. Estos mecanismos de control son previos e independientes de los utilizados durante el funcionamiento y explotación regular del sistema creado (Puigjaner, Serrano y Rubio, 1995).

Establecida la responsabilidad humana del desarrollo, es competencia de éste secuenciar los trabajos, teniendo en consideración las tareas y los recursos destinados a ellas. Como consecuencia de esta ordenación, podrá observarse la existencia de tareas independientes, dependientes, secuenciales y solapadas (Puig 
Torner, 1994: 23-28), según sean las relaciones existentes entre unas y otras, dependiendo de la información de entrada y de salida pertinente a cada una de ellas. Para asegurar la correcta ejecución del proyecto, el cumplimiento de lo planificado, es necesario aplicar medidas de gestión y control. En este sentido, control tomaría el significado de la comprobación del estado del proyecto, y gestión el de las actividades encaminadas a hacer realidad el cumplimiento de lo planificado. La combinación de actividades de ambos tipos permite comprobar el estado del proyecto, y tomar las decisiones oportunas en caso de detectar elementos o factores. Estas comprobaciones periódicas analizan tanto la funcionalidad como la planificación, de forma que se controla, de forma simultánea, la calidad del producto y la capacidad de reacción del proyecto (Puig Torner, 1994: 96-98). Como puede deducirse, el subsistema de control y seguimiento inherente a todo desarrollo de sistemas de información es, a su vez, otro sistema de información que toma como punto de partida la información de gestión generada por la actividad dinámica de desarrollo, para que, una vez procesada ésta, puedan adoptarse decisiones adecuadas, en un proceso de retroalimentación clásico de la teoría de sistemas.

\section{Recapitulación}

El diseño de sistemas de información es una actividad humana en la que el diseñador intenta crear un sistema diseñado, tomando como base un sistema de actividad humana. Se plantea la necesidad de realizar una traslación metódica entre ambos sistemas, que encuentra su mejor expresión en la teoría de sistemas, gracias al principio de isomorfismo. A su vez, el diseño del nuevo sistema objeto se vigila mediante un sistema de control, que se puede identificar con la planificación y gestión del proceso de diseño, desarrollo e implantación. La concepción del sistema de información como abierto, y de la propia organización como un sistema abierto, en continua interacción con el ambiente, adoptado de la teoría de la contingencia, son cuestiones clave tanto en el proceso de desarrollo como en los mecanismos de control de ese desarrollo.

Los mecanismos de control del desarrollo y resultados del proyecto, aunque no han sido objeto prioritario de investigación desde el ámbito de la Documentación, son elementos clave en lo que respecta a resultados, rentabilidad y calidad de los sistemas de información. Los diferentes tipos y métodos utilizados, así como su evolución, pueden rastrearse principalmente en las publicaciones dedicadas a temas de gestión de organizaciones y proyectos. Su integración en el ciclo de vida de los sistemas de información, desde una perspectiva basada en la Documentación, no debe suponer ningún problema, ya que estos mecanismos trabajan y generan información y documentación susceptible de será abor 
dada con los recursos de los documentalistas y especialistas en información de las organizaciones.

\section{Referencias}

Burch, J.G. ; Grudnistky, G. (1992). Diseño de sistemas de información : teoría y práctica. México : Noriega Grupo Megabyte, 1992.

Checkland, P. (1993). Pensamiento de sistemas, práctica de sistemas. México : Megabyte Grupo Noriega, 1993.

Cornellá, A. (1994). Los recursos de información : ventaja competitiva de las empresas. Madrid : McGraw-Hill, 1994.

Lucas, H.C. (1987). Sistemas de Información : análisis, Diseño y Puesta a Punto. Madrid : Paraninfo, 1987.

Martínez López, F.J. (1995) Evolución y paradigma de los Sistemas de Información : Hacia la normalización de la gestión empresarial. Huelva : Universidad de Huelva, 1995.

Martínez Sánchez, J.M.; et al. (1995). Metodología de desarrollo de sistemas de información. Alcalá : Universidad, Servicio de Publicaciones, 1995.

Miguel, A. de ; Piattini, M. (1993). Concepción y diseño de bases de datos : del Modelo E/R al Modelo Relacional. Madrid : RA-MA, 1993

Pablo, I. de, (1989). El reto informático : la gestión de la información en la empresa. Madrid: Pirámide, 1989.

Puig Torner, J. (1994). Proyectos informáticos. Madrid : Paraninfo, 1994.

Puigjaner, R. ; Serrano, J.J. ; Rubio, A. (1995). Evaluación y explotación de sistemas informáticos. Madrid : Síntesis, 1995.

Quang, P.T. ; Gonin, J-J. (1994). Dirección de proyectos informáticos. Barcelona : Gestión 2000, 1994.

Rodríguez, A. ; Márquez, A. (1993). Técnicas de organización y análisis de sistemas. : organización de los servicios informáticos. Madrid : McGraw-Hill, 1993.

Rowley, J. (1994). The Basics of Systems Analysis and Design for Information Managers. London : Clive Bingley, 1994.

Senn, J.A (1992). Análisis y diseño de sistemas de información. México : McGraw-Hill, 1992.

Tramullas, J. (en prensa). Los sistemas de información: una reflexión sobre información, sistema y documentación. // Revista General de Información y Documentación. (En prensa).

Vickery, B.C. ; Vickery, A. (1992). Information Science in Theory and Practice. London : Bowker-Saur, 1992.

Wilson, B. (1993). Sistemas: conceptos, metodología y aplicaciones. México : Megabyte Grupo Noriega, 1993.

Scire. $2: 2$ (jul.-dic. 1996) 121-131 